A Monte Carlo Primer 


\section{A Monte Carlo Primer}

\section{A Practical Approach to Radiation Transport}

Stephen A. Dupree

and

Stanley K. Fraley

Sandia National Laboratories

Albuquerque, New Mexico 
ISBN 978-1-4613-4628-9 ISBN 978-1-4419-8491-3 (eBook)

DOI 10.1007/978-1-4419-8491-3

C2002 Springer Science+Business Media New York

Originally published by Kluwer Academic/Plenum Publishers, New York in 2002

Softcover reprint of the hardcover 1st edition 2002

http://www.wkap.com

$\begin{array}{llllllllll}10 & 9 & 8 & 7 & 6 & 5 & 4 & 3 & 2 & 1\end{array}$

A C.I.P. record for this book is available from the Library of Congress

All rights reserved

No part of this book may be reproduced, stored in a retrieval system, or transmitted in any form or by any means, electronic, mechanical, photocopying, microfilming, recording, or otherwise, without written permission from the Publisher 
To Pat and Huri 


\section{Preface}

The mathematical technique of Monte Carlo, as applied to the transport of sub-atomic particles, has been described in numerous reports and books since its formal development in the 1940s. Most of these instructional efforts have been directed either at the mathematical basis of the technique or at its practical application as embodied in the several large, formal computer codes available for performing Monte Carlo transport calculations. This book attempts to fill what appears to be a gap in this Monte Carlo literature between the mathematics and the software. Thus, while the mathematical basis for Monte Carlo transport is covered in some detail, emphasis is placed on the application of the technique to the solution of practical radiation transport problems. This is done by using the PC as the basic teaching tool.

This book assumes the reader has a knowledge of integral calculus, neutron transport theory, and Fortran programming. It also assumes the reader has available a PC with a Fortran compiler. Any PC of reasonable size should be adequate to reproduce the examples or solve the exercises contained herein. The authors believe it is important for the reader to execute these examples and exercises, and by doing so to become accomplished at preparing appropriate software for solving radiation transport problems using Monte Carlo. The step from the software described in this book to the use of production Monte Carlo codes should be straightforward. However, instead of using these production codes as "black boxes" that must be taken on faith, the reader should be able to understand the functions being performed by the various components of the production software, and in most cases be capable of modifying or expanding it to suit the needs of a particular calculation. 
Mathematical procedures can be expressed in many different ways using high-level computer languages. Fortran is no exception to this generality. Thus there is no "best" way to write a Fortran procedure, and many individual styles, all of which can produce equally valid results, are possible. In our examples we employ executable statements based on Fortran-90, but have often retained the style of older versions of Fortran. We have done this because of our particular experience in using various versions of Fortran. Hopefully readers will be able to follow the constructs easily and to re-cast them into a form compatible with their preferences. To save space we have frequently placed several executable statements in a line of coding.

We have adopted several conventions in the presentation of the text and examples. Fortran subroutines and variable names are designated by single quotation marks. Numerical exponents are expressed either by using the base 10 with a superscript or using the convention of ' + ' or '-' to designate the exponent; i.e., $1.0+5=1.0 \mathrm{E}+5=1.0 \times 10^{5}$. Tabulated results are rounded, with the number of significant digits shown being that which seems appropriate for the example in hand. All calculations that involve using computed results to obtain additional results use the values prior to rounding. Therefore, if the derived results are reproduced using the rounded values presented in the tables the numbers obtained may differ from those cited in the text. Because compilers and execution speeds differ, the user should not expect to reproduce the problem run times cited in this book. However, the relative efficiencies of a series of calculations should be similar regardless of the compiler and computer used.

We gratefully acknowledge the assistance of our friends and colleagues who reviewed the manuscript. Kevin O'Brien and Jim Renken provided thorough and detailed reviews of the entire text, and Eleanor Walther reviewed the appendix. In all cases their comments were well considered and informative, and they saved us from numerous omissions, obfuscations, and errors. Special thanks go to Len Connell and Tom Laub, who not only reviewed the text but also devoted considerable effort to checking and using the Monte Carlo routines. Long before the manuscript was completed Len Connell was the first user of the probabilistic framework code. Tom Laub provided independent solutions and numerous corrections to the example problems. The time and efforts of these reviewers are much appreciated and this book has greatly benefited from their support. Needless to say, any errors remaining in the text are the responsibility of the authors.

Stephen A. Dupree

Stanley K. Fraley

Albuquerque, NM

July 2001 


\section{Contents}

\section{Chapter 1. Introduction}

1.1 The Monte Carlo Method

1.2 The Evaluation of Random Processes

Example 1.1 Predicting the average outcome of a physical process 6

Example 1.2 Another Monte Carlo estimate of $\pi \quad 11$

1.3 Monte Carlo Evaluation of Definite Integrals 14

Example 1.3 Evaluation of a definite integral using Monte Carlo

Exercises

\section{Chapter 2. Monte Carlo Sampling Techniques}

2.1 Probability Theory and Statistics

2.1.1 Random Variables and Sample Spaces

2.1.2 Distributions

2.2 Sampling

2.2.1 Sampling from the Inverse of the Cumulative Distribution Function

Example 2.1 Uniform sampling inside a sphere 28

2.2.2 The Rejection Technique 30

2.3 Means and Variances $\quad 32$

2.4 Estimations of Means and Variances 35

Example 2.2 Calculation of mean and variance of a distribution 
2.5 Introduction to Variance Reduction Techniques

Example 2.3 Variance reduction by repeated samples

2.5.1 Stratified Sampling

Example 2.4 Use of stratified sampling to evaluate a definite integral

2.5.2 Biased Sampling Schemes

Example 2.5 Biasing a Monte Carlo estimate of a definite integral

Exercises

\section{Chapter 3. Monte Carlo Modeling of Neutron Transport}

3.1 Introduction

3.2 Neutron Interactions and Mean Free Path 57

3.3 Neutron Transport

3.4 A Mathematical Basis for Monte Carlo Neutron Transport

3.5 Monte Carlo Modeling of Neutron Motion

Example 3.1 Monoenergetic point source with isotropic scattering

Example 3.2 Self-attenuation in a spherical source of gamma rays

Example 3.3 Beam of neutrons onto a slab shield

3.6 Particle Flight Path in Complex Geometries

Example 3.4 Mean distance to the next collision 81

3.7 Multi-Region Problems

Example 3.5 Two-region slab with a void

Exercises

\section{Chapter 4. Energy-Dependent Neutron Transport}

4.1 Elastic Scattering of Neutrons

Example 4.1 Average number of collisions to thermalize neutrons

4.2 Transformation of Post-Collision Direction to Laboratory System

Example 4.2 Average direction of travel after two collisions

4.3 Energy-Dependent Cross Sections

Example 4.3 Neutron slowing down and Fermi age in water 
Chapter 5. A Probabilistic Framework Code

5.1 Introduction to PFC

5.2 Problem Definition in PFC

5.2.1 Problem Geometry and Tracking

5.2.2 Additional Input and Array Initialization

122

5.3 The Random Walk in PFC

124

5.4 Computing the Response

Exercises

\section{Chapter 6. Variance Reduction Techniques}

6.1 Introduction

139

6.2 Source Biasing

Example 6.1 Leakage of particles from a slab

6.3 Survival Biasing

Example 6.2 Particles passing through a slab

151

6.4 Russian Roulette

154

Example 6.3 Russian roulette in the slab problem of Example 6.2

6.5 Splitting

Example 6.4 Slab problem with splitting and Russian roulette

Example 6.5 Transmission through a slab with exponential transform

Exercises

\section{Chapter 7. Monte Carlo Detectors}

7.1 Introduction

7.2 The Next-Event Estimator

Example 7.1 Next-event flux estimates in an isotropic scattering material

7.3 Volumetric Flux Detectors

Example 7.2 Collision-density and track-length flux estimates

7.4 Surface-Crossing Flux Estimator 
Example 7.4 Expectation surface-crossing flux estimates

7.6 Time-Dependent Detectors

Example 7.5 Time-dependence of the flux from a point isotropic source

Example 7.6 Time dependence in neutron slowing down

\section{Chapter 8. Nuclear Criticality Calculations with Monte Carlo}

8.1 Multiplying Assemblies

8.2 The Generation Method

Example 8.1 Criticality in a homogeneous sphere by ratio of generations

8.3 The Matrix Method

Example $8.2 \mathrm{k}$ for a homogeneous sphere using matrix method

8.4 Combination of Generation and Matrix Methods

Example 8.3 Multi-generation matrix calculation

8.5 Criticality Calculations Using Multigroup Cross Sections 258 Exercises

Example 8.4 Critical mass of the Godiva assembly 259

\section{Chapter 9. Advanced Applications of Monte Carlo}

9.1 Correlated Sampling

Example 9.1 Generating correlated strings of random numbers

Example 9.2 Sensitivity of the number of particles passing through a slab to the thickness of the slab

9.2 Adjoint Monte Carlo

Example 9.3 Detector response function using adjoint transport

Example 9.4 A point-detector response using adjoint transport

9.3 Neutron Thermalization

Example 9.5 Neutron spectrum in thermal equilibrium 
Appendix

Random Number Generators

Bibliography

329

Index 